\title{
O DESIGN COMO DIFERENCIAL ESTRATÉGICO NA CONSTRUÇÃO DE DASHBOARDS
}

\author{
Thiago Pessato \\ UFSC \\ thiagopessato@gmail.com \\ Mônica Stein \\ UFSC \\ moni_stein@yahoo.com.br
}

\section{Resumo}

Este artigo visa demonstrar o que são dashboards e como eles são desenvolvidos atualmente, enfatizando o papel que a gestão de design pode desempenhar no seu processo de construção. Pretende ainda posicionar o design não somente nas questões visuais e de interface, mas como agente de inovação, sugerindo uma abordagem desde o início do processo de desenvolvimento até a entrega destas ferramentas aos usuários. Ressalta-se que, para facilitar o entendimento dos conceitos expostos neste artigo, o termo dashboard tem um significado mais amplo e refere-se a todo o tipo de aplicação de análise de dados.

Palavras-chave: Gestão de Design, Design Estratégico, Dashboards, Ferramentas Analíticas, Business Intelligence

\section{ABSTRACT}

This article aims to demonstrate what dashboards are and how they are developed nowadays, emphasizing the role that design management can play in its construction process. It also aims to position the design not only on visual and interface issues, but primarily as an agent of innovation, suggesting an approach from the beginning of the development process to delivery of these tools to users. It is highlighted that, to facilitate understanding of the concepts presented in this article, the term dashboard has a broader meaning and refers to any type of data analysis application.

Keywords: Design Management, Strategic Design, Dashboards, Analytical Tools, Business Intelligence

\section{INTRODUÇÃO}

A evolução tecnológica tem-se revelado intensa nas últimas décadas, onde todo tipo de dados, sejam quantitativos ou qualitativos, são disponibilizados, muitas vezes 
instante a instante, através das diversas tecnologias existentes, principalmente por meio da Internet. A utilização de softwares, através dos quais são criadas as interfaces para manipular esses dados, tem sido fundamental para disponibilizá-los visualmente, cabendo ao design ${ }^{1}$ organizá-los em sistemas compreensivos.

Na esfera corporativa, as organizações tem gerado cada vez mais dados sobre seus negócios através da adoção de softwares de gestão empresarial, como as soluções de ERP (Enterprise Resource Planning), CRM (Customer Relationship Managament) e tantos outros programas complementares para apoio à gestão de diversas áreas como financeira, administrativa, de recursos humanos e processos.

Consequetemente, o uso de aplicações de análise de dados, como os dashboards, tem permitido potencializar o controle para que ações corretivas sejam adotadas quando necessário. Eles são compostos por indicadores de performance, como por exemplo o painel de dados de um automóvel, que apresenta ao condutor informações como velocidade, rotação e temperatura do motor, nível do óleo, dentre outros. $\mathrm{Na}$ área de negócios, dashboards são o conjunto dos principais indicadores que permitam o gerenciamento de alguma operação, função, processo ou estratégia.

Para exemplificar, na figura 01, à esquerda, pode-se ver o dashboard de um automóvel. Nele o usuário tem acesso às informações mais relevantes sobre seu funcionamento, de maneira rápida e visível. À direita, percebe-se um dashboard ${ }^{2}$ operacional e acessível através da internet, em que um gerente pode monitorar e analisar o desempenho e carga de trabalho de uma equipe de engenheiros em campo.
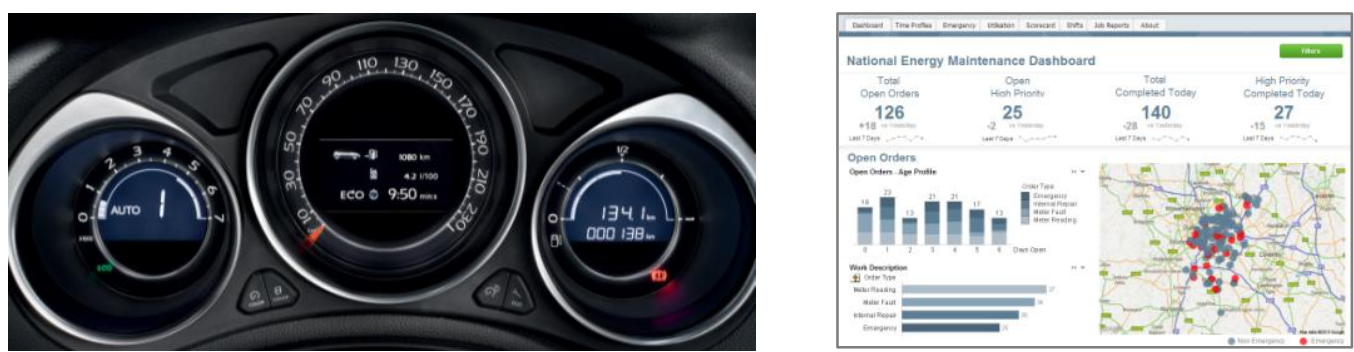

Figura 01 - Dashboards de um automóvel e de ocorrências de manutenção e fornecimento de energia ${ }^{3}$.

\footnotetext{
${ }^{1}$ Salienta-se que, embora importantes e pertinentes ao universo discutido, questões como usabilidade e interface gráfica não serão discutidas por não serem o foco deste artigo.

${ }^{2}$ A expressão "Dashboard" e "Painel de Controle" serão utilizadas como sinônimos ao longo deste artigo.

${ }^{3}$ Fonte:<http://ap.demo.qlikview.com/detail.aspx?appName=Workforce\%20Management.qvw > Acesso em: jan. 2013. < http://grafster.net/carstar/14/dashboard-citroen-ds4-2012-car-photo13_size0.html > Acesso em: mar. 2014.
} 
Estas ferramentas são elaboradas com o objetivo de exibir variados tipos de informação com o máximo de exatidão e detalhamento possíveis, porém ainda sem a devida preocupação a respeito do uso concreto e dos benefícios que estes dados podem trazer para as organizações. Consequentemente, este fato abre um campo de pesquisa para a exporação do design não apenas como um processo voltado a criação de interfaces, mas em um âmbito anterior, orientado ao planejamento estratégico dos dashboards.

Logo, este artigo pretende demonstrar como o design pode colaborar no processo de construção dessas ferramentas de modo a torná-las eficientes para a gestão estratégica das organizações.

\section{DASHBOARDS E SEU CONTEXTO NA ÁREA DE TI}

Segundo Few (2006), pesquisador de maior destaque na área ${ }^{4}$, um dashboard é "um display visual das informações mais importantes necessárias para alcançar um ou mais objetivos, consolidados e organizados em uma única tela para que a informação possa ser monitorada em um piscar de olhos."

Dashboards e demais aplicações de análise de dados estão inseridos em um segmento da Tecnologia da Informação chamado de Business Intelligence. Estas aplicações são construídas com base em Ferramentas Analíticas.

O conceito de Business Intellingence ${ }^{5}$ foi estabelecido na década de 1980 pelo Gartner Group" que o define como "um termo abrangente que inclui os aplicativos, infraestrutura, ferramentas e melhores práticas que permitem o acesso e a análise de informações para melhorar e otimizar decisões e desempenho." ${ }^{7}$

Os esquemas abaixo demonstram a correlação entre as partes, tendo-se como ponto de referência o segmento de Tecnologia da Informação (TI), assim como o Conceito de $\mathrm{BI}$ (Business Intelligence)
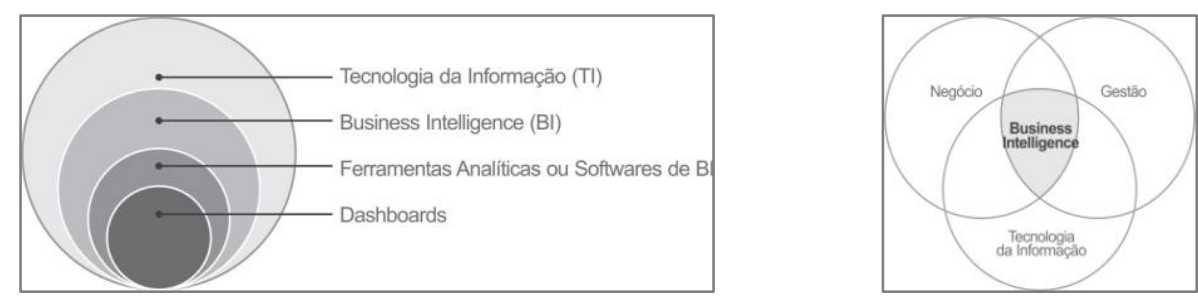

\footnotetext{
${ }^{4}$ Este artigo embasa-se em Stephen Few para a maioria das definições/discussões sobre dashboard, pela falta de bibliografia pertinente adicional na área, sendo este o autor de maior presença e destaque até o momento.

5 Os termos equivalentes em português podem ser "Inteligência Empresarial", "Inteligência de Negócios" ou "Inteligência Competitiva".

${ }^{6}$ Empresa norte-americana fundada em 1979 - líder mundial em pesquisa e consultoria em Tecnologia da Informação.

${ }^{7}$ Fonte: < http://www.gartner.com/it-glossary/business-intelligence-bi/ > (Acesso em: jan. 2013)
} 
Figura 03 - A esquerda o contexto dos dashboards e demais aplicações de análise de dados. A direita demostra-se o Business Intelligence ou "BI", que atua no centro das organizações e permite a leitura de informações vitais para o controle do seu desempenho. Fonte: Elaborado pelos autores, com base na pesquisa realizada

Portanto, os dashboards são parte integrante e fundamental da área de Business Intelligence, sendo eventualmente chamados de "Ferramentas de Bl". Abaixo é possível uma comparação do grau de esforço entre a análise de dados tradicional (cenário 1) e da abordagem através de ferramentas de $\mathrm{BI}^{8}$ (cenário 2 ).

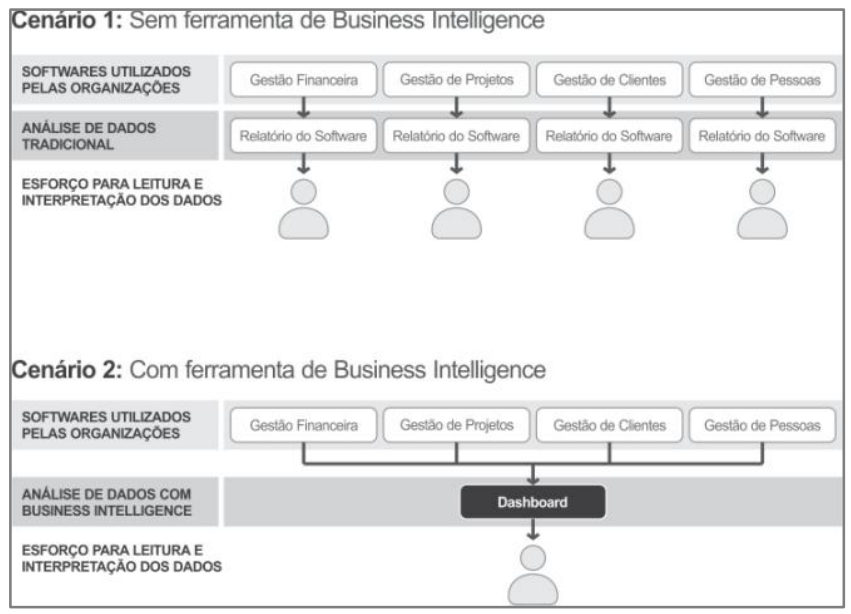

Figura 04 - Cenário hipotético de uma organização que utiliza diversos softwares de apoio à gestão. Fonte: Elaborado pelos autores, com base na pesquisa realizada

No cenário 1 o esforço para interpretação dos relatórios emitidos por cada uma dos softwares de gestão é proporcional ao número de ferramentas utilizadas. Já no cenário 2, a utilização de ferramenta de $\mathrm{BI}$ através de dashboards permite consolidar os mesmos relatórios individuais (do cenário 1) em uma única interface, reduzindo o esforço para leitura. Permite ainda gerar gráficos e métricas personalizados que não sejam nativas nos relatórios individuais do cenário 1.

\subsection{Categorias de Dashboards}

Os painéis de controle são compostos por gráficos que permitem monitorar diversos tipos de dados e dar suporte a quase todo o tipo de objetivo necessário para os negócios de uma instituição. Segundo Few (2006), há várias maneiras de categorizar estas aplicações em diversos tipos. Podem ser estratégicas, analíticas, ou operacionais, e as características do design no que tange à sugestão de organização variam para dar suporte às necessidades de cada categoria: estratégica; analítica; e operacionais.

\footnotetext{
${ }^{8}$ Softwares de Business Intelligence (BI) têm como principal benefício para as organizações a velocidade na extração e exibição de dados provenientes de diversos softwares de gestão, permitindo uma rápida análise sobre estes diferentes volumes de dados.
} 


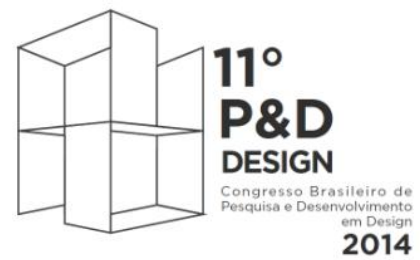

Gramado - RS

De 29 de setembro a 2 de outubro de 2014

a. Dashboards para fins estratégicos: Para Few (2006, p. 41), "O uso primários de dashboards nos dias de hoje é para propósitos estratégicos (...) oferecem uma rápida visão que os tomadores de decisão precisam para monitorar a saúde e as oportunidades de um negócio (...)".

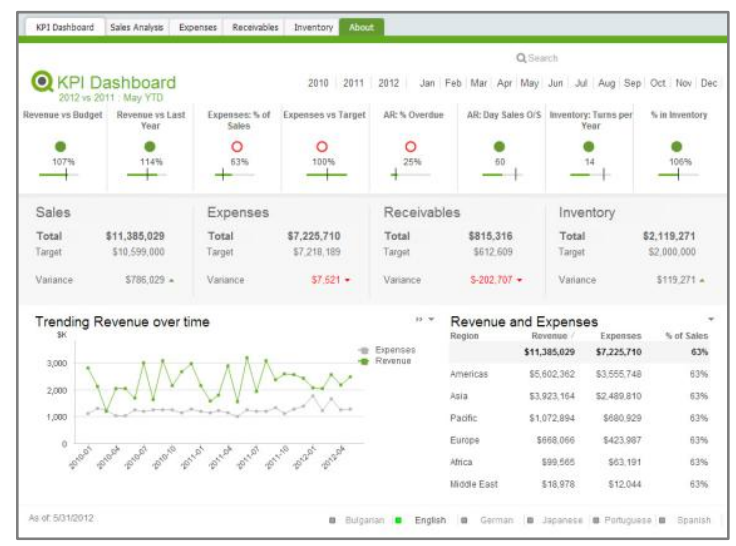

Figura 05 - Exemplo de Dashboard estratégico ${ }^{9}$

No que tange a visualização de dados, Few (2006, p. 41) salienta que "(...) mecanismos extremamente simples de visualização funcionam melhor para este tipo de dashboard(...)".

b. Dashboards para fins analíticos: Para fins analíticos, Few (2006, p. 41) define mais sofisticação para as mídias de exibição, para que os analistas possam examinar melhor dados complexos e relacionamentos. "(...) Dashboards analíticos devem suportar interações com os dados, como aprofundamentos em camadas detalhadas (...) não apenas para ver o que está acontecendo, mas para examinar as causas.

Este tipo de dashboard, como demonstrado abaixo (figura 06) se assemelha visualmente com o estratégico, porém apresenta mais opções de filtros e interações, permitindo que a análise seja feita em níveis mais detalhados do que o primeiro.

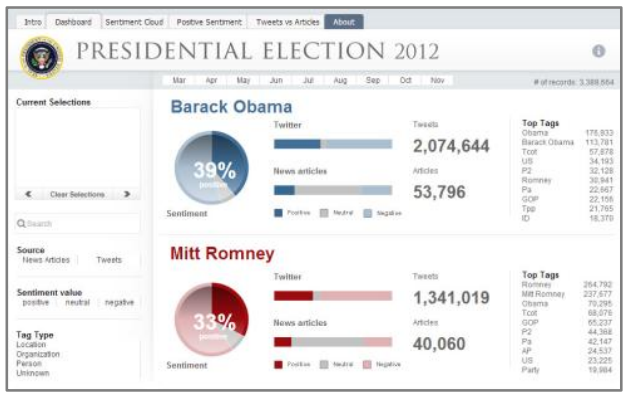

\footnotetext{
${ }^{9}$ Fonte: < http://ap.demo.qlikview.com/detail.aspx?appName=Executive\%20Dashboard.qvw > Acesso em: fev. 2013.
} 
Figura 06 - Exemplo de Dashboard analítico ${ }^{10}$

c. Dashboards para fins operacionais: Few (2006, pg 41) justifica que dashboards que monitorem operações devem "(...) manter consciência das atividades e eventos que estão mudando constantemente e podem demandar atenção e resposta (...).

A figura abaixo demonstra um painel operacional que monitora os vôos atrasados que saem do Reino Unido. As manchas em verde, amarelo e vermelho indicam o nível de atraso dos voos saindo da cidade de Birmingham.

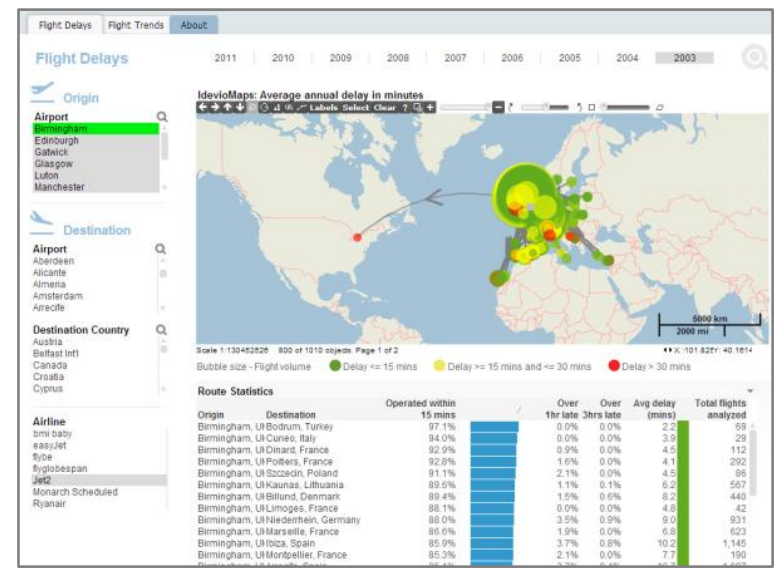

Figura 07 - Exemplo de Dashboard operacional ${ }^{11}$

O quadro abaixo permite que se perceba uma lista de variáveis que podem servir para estruturar taxonomias de ferramentas analíticas:

\begin{tabular}{|l|l|l|l|}
\hline Função & Tipo de dados & Domínio dos dados & Frequência de atualização \\
\hline Estratégica & Quantitativo & Vendas & Mensal \\
\hline Analítica & Não-quantitativo & Finanças & Semanal \\
\hline Operacional & & Marketing & Diário \\
\hline & & Produção & Por hora \\
\hline Espaço de dados & Recursos Humanos & Em tempo real \\
\hline Empresarial & Interatividade & Mecanismos de visualização & Funcionalidade de portal \\
\hline Departamental & Estática & Primariamente gráfica & Conduz para dados adicionais \\
\hline Individual & Interativa & Primariamente textual & Sem funcionalidades de portal \\
\hline
\end{tabular}

Quadro 01 - Possíveis classificações para Dashboards. Fonte: FEW (2006 - p. 39-40)

\subsection{Infográficos: Data Visualization x Data Art}

Para ter uma leitura mais fácil e atraente, os painéis de controle lançam mão de gráficos de análise e outros objetos, chamados de objetos de data visualization ${ }^{12}$. São

\footnotetext{
10 . Fonte: < http://ap.demo.qlikview.com/detail.aspx?appName=2012\%20Presidential\%20Election.qvw $>$ Acesso em: mar. 2014.

${ }^{11}$ Fonte: < http://ap.demo.qlikview.com/detail.aspx?appName=Flight\%20Delays.qvw> Acesso em: mar. 2014.
} 
eventualmente confundidos com data art, porém diferem quanto ao seu objetivo principal: enquanto data visualization tem como objetivo principal apresentar dados através de imagens de forma que sejam o mais simples e de rápido entendimento possível, data art refere-se a objetos que tenham a função de atrair visualmente o usuário, onde a interpretação dos dados estão em segundo plano.
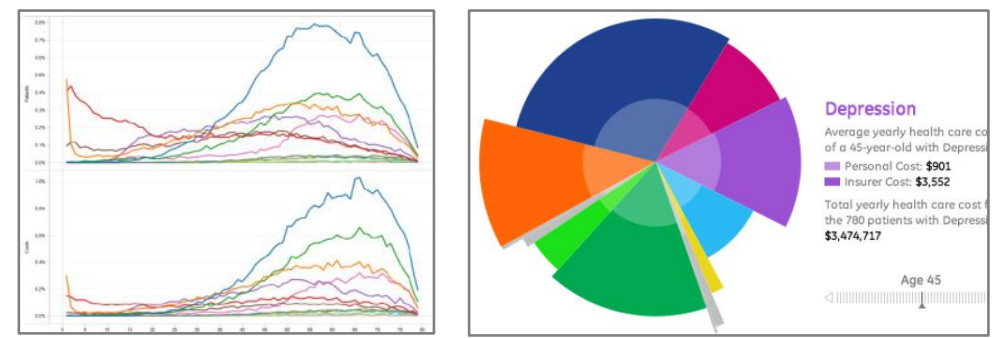

Figura 08: Na esquerda um "Data Visualization", onde os gráficos de linhas demonstram dados quantitativos de forma clara e fácil. Na imagem da direita um "Data Art", em que o gráfico de "pizza" tem o objetivo de chamar a atenção do usuário, porém a interpretação dos dados é prejudicada pelo design atribuído apenas a visualização do objeto. Fonte: FEW (2010)

Gelman e Unwin (2012, p. 2), reforçam a importância da atuação dos designers na construção deste tipo de objeto informativo: "Nos preocupamos com o fato de que os designers de gráficos não-estatísticos não estejam tão focados em transmitir informação, onde a beleza de muitas imagens profissionalmente produzidas, paradoxalmente, não atinjam o objetivo de um melhor entendimento dos dados."

Os autores utilizam em seu artigo os termos "statistical graphics" e "information visualization (Infovis)" para se referir a "data visualization" e "data art", respectivamente. A diferença fica evidente ao comparar seus objetivos: "Em termos estatísticos, os analistas de dados e estatísticos estão interessados em encontrar efetividade e meios precisos de representar os dados, sejam dados brutos, estatísticas ou análises modelo." Ainda afirmam que "(...) Em termos de Infovis, cientistas da computação e designers estão interessados em conseguir a atenção dos leitores, contando a eles uma história (...)" Gelman e Unwin (2012, p. 2).

Logo, cabe ao designer fazer o melhor uso das abordagens textual e gráfica em um dashboard. Numa abordagem textual, os dados são exibidos em forma de números e textos, não devendo ser utilizada de maneira moderada, já que os textos requerem leitura, envolvendo processamento verbal do cérebro, que é mais lento do que o processamento visual. Abaixo apresentam-se quatro grupos de dados com coordenadas muito semelhantes mas que, se representadas através de gráficos, demonstram variações significativas e facilmente compreendidas. O estudo foi desenvolvido pelo estatístico Francis Anscombe em 1973 para provar a eficiência da visualização de dados através de gráficos em comparação com a abordagem textual.

\footnotetext{
${ }^{12}$ Termo em inglês para "visualização de dados"
} 


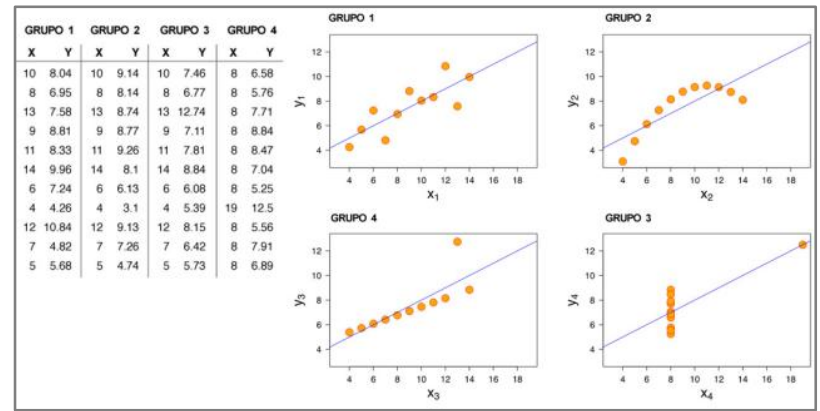

Figura 09: Quarteto de Anscombe. Fonte: Graphs in Statistical Analysis (Francis Anscombe - 1973)

\subsection{Gestão de Design no processo de desenvolvimento de dashboards}

Embora o termo design seja frequentemente relacionado apenas a questões estéticas ou mesmo a "desenho" ou "projeto", ele engloba também a ideia de resolução de problemas através de conceitos como o design thinking ${ }^{13}$. Esta visão de design, e principalmente o reconhecimento dos gerentes de negócios quanto à importância dessa abordagem de gestão, contribui para a sua inserção em áreas que ultrapassam os limites da atuação tradicional do designer.

$\mathrm{Na}$ gestão de uma empresa, pensar como designer mostra-se útil para identificar potenciais caminhos futuros, estabelecer pontes com os clientes e simplificar o modelo de negócio (GOMES, 2010). Para MOZOTA (2003, p. 71), gestão de design "(...) é a implementação do design como um programa formal de atividades dentro da empresa pela comunicação da relevância do design para as metas organizacionais de longo termo e coordenar recursos de design ao nível de atividade empresarial para atingir objetivos organizacionais (...).

No que refere-se aos dashboards, o design está relacionado diretamente à gestão, visto que é através destas ferramentas que as organizações conseguem ter insights sobre o seu mercado e atuação. Dezenas de softwares de Bl foram desenvolvidos para atender a esta demanda crescente por informação. Apesar do cenário favorável, pouco esforço e atenção são direcionados para questões de design destas ferramentas, desde o real uso que delas é feito até a questões técnicas de data visualization.

As empresas que desenvolvem aplicações de análise de dados costumam envolver equipes multidisciplinares compostas por gestores comerciais, gestores de projetos, desenvolvedores analistas (de sistemas ou estatísticos) e, mais recentemente, designers. Como em qualquer prestação de serviço, o desenvolvimento

\footnotetext{
${ }^{13}$ Refere-se à maneira do designer de pensar, onde busca-se formular questionamentos através da compreensão dos problemas, ou seja, são formuladas perguntas a serem respondidas a partir das informações coletadas durante a observação.
} 
envolve também o cliente através dos diretores, gestores, equipe de tecnologia da informação e usuários da ferramenta que será entregue.

Os desenvolvedores analistas, em sua maioria, ocupam-se apenas em exibir os dados corretos (os mesmos dados extraídos dos softwares de gerenciamento dos clientes) na interface do dashboard, do que propriamente com a finalidade e eficiência no uso destes objetos.

É comum estes analistas desenvolverem a interface seguindo uma lista de requisitos e de indicadores de performance ${ }^{14}$ levantadas pelo próprio cliente. Se por um lado este checklist facilita e guia o trabalho do analista durante o desenvolvimento da aplicação, por outro ele limita as possibilidades de solução e induz o cliente a ter as mesmas visões as quais já está acostumado. Em suma, embora em geral os clientes acreditem saber exatamente o que é melhor para a sua empresa em termos de análise, é justamente por estarem imersos nas questões do próprio negócio que faz com que muitas vezes as visões possíveis através de dashboards possam não agregar um diferencial importante para a gestão da instituição.

O designer, por sua vez, ao invés de se preocupar com a exatidão das informações, busca identificar inicialmente o principal objetivo dos clientes ao usarem uma ferramenta analítica. Com base neste briefing e no entendimento do negócio do cliente, propõe não somente os objetos de análise e métricas mais adequados, mas também possíveis infográficos que possibilitem um entendimento mais claro dos dados que se está exibindo.

A visão e atuação do designer acaba por impactar diretamente nas questões estratégicas da empresa, visto que é através da interface gráfica desenvolvida que o cliente terá uma visão clara do desempenho do seu negócio.

O diagrama a seguir resume genericamente as principais fases de um projeto de desenvolvimento de dashboard:

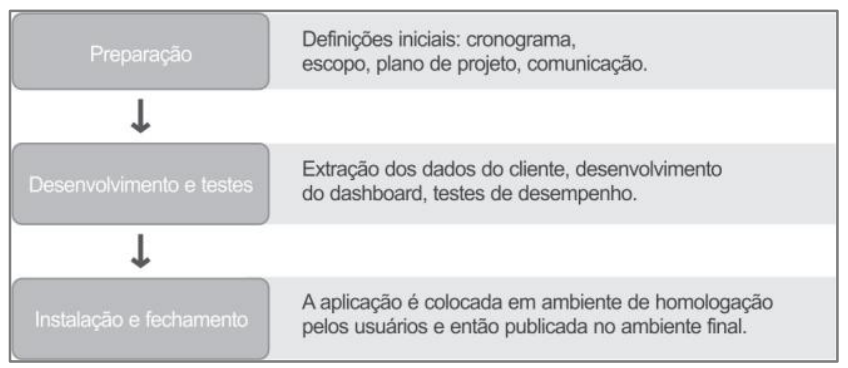

Figura 10: Esquema genérico de processo de desenvolvimento de dashboards Fonte: Elaborado pelos autores, com base na pesquisa realizada

\footnotetext{
${ }^{14}$ Também chamados de KPI (Key Performance Indicator)
} 
A maioria dos softwares de $\mathrm{BI}$ permitem uma abordagem de processo mais curta, o que é benéfico em termos de prazo, porém nem sempre resulta na melhor qualidade do produto entregue. A fase de desenvolvimento e testes apontadas no quadro acima, por exemplo, engloba questões fundamentais quanto à metodologia de desenvolvimento de softwares, como design e integração. Como não há formalização destas etapas e a saída da fase de "Desenvolvimento e testes" é a aplicação já finalizada, há uma tendência natural à supressão da etapa de design. Não raro, a preocupação com o design acaba por surgir somente no final desta fase, onde o seu papel principal é de organizar visualmente o dashboard.

Metodologias de desenvolvimento que considerem o design (ou "projeto") como etapa distinta podem acarretar melhores resultados, como o modelo em cascata de desenvolvimento de software:

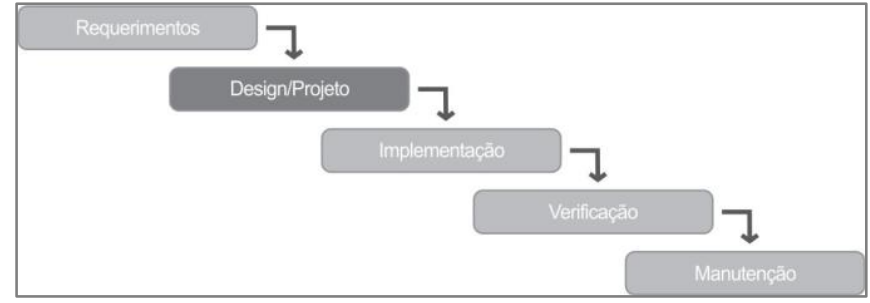

Figura 11: Modelo "Waterfall" (cascata) de desenvolvimento de software é amplamente utilizado e considera o design como importante etapa. Fonte: Elaborado pelos autores, com base na pesquisa realizada ${ }^{15}$

A importância da inserção do design no início do processo de desenvolvimento de dashboards é fundamental para a definição de questões como: objetivo principal, perfil da audiência (usuários), familiaridade da audiência com ferramentas de análise, dashboard como ferramenta de endomarketing (grau de importância já que em geral os dashboards são utilizados por colaboradores das instituições), requisitos de entrega (dispositivos móveis, etc.). A figura abaixo demonstra genericamente o processo de desenvolvimento de websites. A similaridade estratégica permite sua adoção em parte também no desenvolvimento de ferramentas de BI:

\footnotetext{
${ }^{15}$ (modificado a partir de http://en.wikipedia.org/wiki/Waterfall_model)
} 


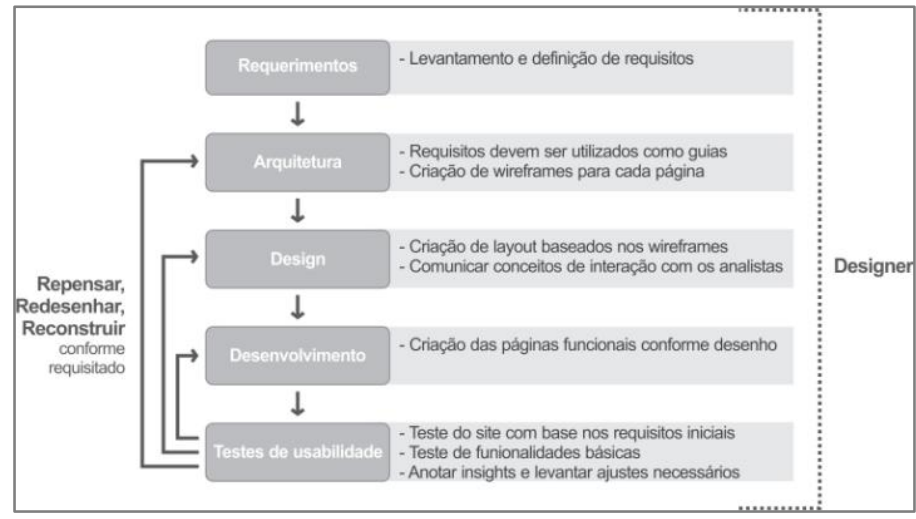

Figura 12: Fluxograma de desenvolvimento de websites. A atuação do designer deve se dar em todas as etapas. Fonte: Elaborado pelos autores, com base na pesquisa realizada

Embora fundamental no início do processo, a atuação do designer na criação de dashboards não se limita às questões de requisitos e objetivos gerais atrelados ao briefing. Seu conhecimento nas questões de uso adequado de cores, conceitos de diagramação, hierarquia de informação, gestalt, dentre outros é primordial no trabalho em conjunto com os analistas desenvolvedores em busca da melhor solução.

\section{POSSIBILIDADES FUTURAS ENTRE DESIGN E DASHBOARDS}

Diante do exposto, pode-se pressupor que a construção de painéis de controle atravessa uma fase de amadurecimento que a própria internet e seus websites ultrapassaram décadas atrás. No começo, engenheiros, programadores e analistas de sistemas trabalharam para viabilizar a utilização de interfaces através da rede mundial de computadores. A preocupação com a experiência de uso e o visual destas interfaces era menor e só amadureceu com a inclusão formal de designers durante as fases de desenvolvimento. Assim como no caso dos websites, a construção de dashboards está progredindo no mesmo caminho, onde a atuação do designer pode ser central e acompanhar as principais fases de desenvolvimento destas ferramentas, cabendo ao mesmo, neste distinto cenário, enfrentar o desafio de entender os detalhes, dinâmicas e pormenores de cada negócio ou projeto, a fim de poder propor as melhores soluções visuais para a correta interpretação dos dados.

Contudo, não se pretende aqui propor um processo novo, mas sim salientar a importância do design relacionado a sua inserção desde o início do projeto/concepção, na tentativa de desvinculá-lo de interpretações simplistas que o releguem apenas a solucionar questões "estéticas" no final do processo. Para projetos de desenvolvimento de dashboards que precisam ser mais rápidos, seja em função de orçamento e/ou prazo curtos, a atuação do designer pode se dar de forma pontual, com o objetivo de trabalhar exclusivamente com data visualization e interface gráfica, mas ainda assim sem perder o foco nas respostas que o dashboard deve oferecer aos usuários. Porém, não se intenciona colocar a figura do designer isolada dos demais 
profissionais em virtude da sugestão da inclusão de etapas formais de design durante o processo, pois é sabido que a interdisciplinaridade e o trabalho em conjunto entre os profissionais das diversas áreas envolvidos é ponto crucial e fator de sucesso. Por sua formação e visão holística, o designer pode contribuir de forma decisiva no desenvolvimento de dashboards, não apenas através de interfaces gráficas mais eficientes, mas também em questões estratégicas quanto à definição do que será exibido dentro da aplicação, com o intuito de permitir que se monitore o desempenho de fatores vitais para a diferenciação das organizações.

\section{REFERÊNCIAS}

ADLER, Isabel; LUCENA, Brenda; RUSSO, Beatriz, VIANNA, Maurício; VIANNA, Ysmar. Design Thinking: Inovações em Negócios. MJV Press, 2011. Disponível em:

<http://livrodesignthinking.com.br/download/livro_dt_MJV.pdf>

ANSCOMBE, Francis. Graphs in Statistical Analysis. Disponível em:

<http://www.sjsu.edu/faculty/gerstman/StatPrimer/anscombe1973.pdf>. Acesso em jan.

2013.

FEW, Stephen. Data Art and Data Visualization. Disponível em:

$<$ http://www.perceptualedge.com/blog/?p=1245>. Acesso em set. 2012.

FEW, Stephen. Information Visualization, Design, and the Arts Collision or Collaboration?.

Disponível em:

$<$ http://www.perceptualedge.com/articles/visual_business_intelligence/information_visualiza tion_and_art.pdf>. Acesso em jan. 2013.

FEW, Stephen. Information Dashboard Design - The Effective Visual Communication of Data. Sebastopol: O'Really Media, 2006.

GELMAN, Andre; UNWIN, Antony. Infovis and Statistical Graphics: Different Goals, Different Looks. Disponível em:

$<$ http://www.stat.columbia.edu/ gelman/research/published/vis14.pdf $>$. Acesso em dez.

2012.

GOMES, Adriana. Dossiê Pensamento de design - Quando menos é mais. HSM Management 79 março-abril 2010. Disponível em:

<http://www.ngd.ufsc.br/files/2012/04/designHSM_Dossie2.pdf>

LIEDTKA, Jeanne. Se os executivos pensassem como designers... HSM Management 62 maiojunho 2007. Disponível em: <http://www.ngd.ufsc.br/files/2012/04/executivos-

designers1.pdf>

MARTINS, Rosane Fonseca de Freitas; MERINO, Eugenio Andrés Diaz. A gestão de design como estratégia organizacional. 2. Ed. Londrina: Eduel; Rio de Janeiro: Rio Books, 2011.

MOZOTA, Brigite Borja de. Design Management. Using to build brand value and corporate innovation. New Yourk: Allworth Press, 2003.

QLIKTECH INTERNATIONAL AB. QlikView Technical Brief: How to improve your design process working with QlikView. Disponível em:

<http://community.qlikview.com/servlet/JiveServlet/download/38-

43272/Technical\%20Paper\%20-

\%20How\%20To\%20Improve\%20Your\%20Design\%20Process\%20Working\%20With\%20Qlikview .pdf>. Acesso em jun. 2012. 